\title{
A systematic comparison of fibroblasts derived from postmortem human dura mater versus dermal epithelium for neurodegenerative disease modeling
}

Authors

5 Andrea R. Argouarch ${ }^{1}$, Celica G. Cosme ${ }^{1}$, Kristle Garcia ${ }^{2,3}$, Christian I. Corrales ${ }^{1}$, Alissa L. 6 Nana $^{1}$, Anna M. Karydas ${ }^{1}$, Salvatore Spina ${ }^{1}$, Lea T. Grinberg ${ }^{1,4}$, Bruce Miller ${ }^{1}$, Hani Goodarzi ${ }^{2,3}$, 7 William W. Seeley ${ }^{1,4}$, and Aimee W. Kao ${ }^{1 *}$

\section{Affiliations}

$10{ }^{1}$ Memory and Aging Center, Weill Institute for Neurosciences, Department of Neurology, 11 University of California, San Francisco, San Francisco, CA, 94158, USA

$12{ }^{2}$ Department of Biochemistry and Biophysics, University of California San Francisco, San

13 Francisco, CA, 94158, USA

$14{ }^{3}$ Department of Urology, University of California San Francisco, San Francisco, CA, 94158, 15 USA

$16{ }^{4}$ Department of Pathology, University of California San Francisco, San Francisco, CA, 94158, 17 USA

Andrea R. Argouarch - andrea.argouarch@ucsf.edu

Celica G. Cosme - ccelica94@gmail.com

21 Kristle Garcia - kristle.garcia@ucsf.edu

22 Christian I. Corrales - khriscor2008@gmail.com

23 Alissa L. Nana - alissa.nanali@ucsf.edu

24 Anna M. Karydas - akarydas@me.com

25 Salvatore Spina - salvatore.spina@ucsf.edu

26 Lea T. Grinberg - lea.grinberg@ucsf.edu

27 Bruce Miller - bruce.miller@ucsf.edu

28 Hani Goodarzi - hani.goodarzi@ucsf.edu

29 William W. Seeley - bill.seeley@ucsf.edu

30 Aimee W. Kao-aimee.kao@ucsf.edu

$31 *$ Corresponding author - Aimee W. Kao. Correspondence to: aimee.kao@ ucsf.edu

\section{Keywords}

Human dura mater, dermal epithelium, dural cells, dermal fibroblasts, postmortem tissue, 


\section{ABSTRACT}

38 Patient-derived cells hold great promise for precision medicine approaches in human health.

39 Fibroblast cells have been a major source of human cells for reprogramming and differentiating

40 into specific cell types for disease modeling. Such cells can be isolated at various stages during

41 life (presymptomatic, symptomatic, and postmortem) and thus can potentially be used to model

42 different phases of disease progression. In certain circumstances, however, tissues are not

43 collected during life and only postmortem tissues are the only available source of fibroblasts.

44 Fibroblasts cultured from postmortem human dura mater of individuals with neurodegenerative

45 diseases have been suggested as a primary source of cells for in vitro modeling of

46 neurodegenerative diseases. Although fibroblast-like cells from human and mouse dura mater

47 have been previously described, their utility for reprogramming and direct differentiation

48 protocols requires further characterization. In this study, cells derived from dermal biopsies

49 performed in living subjects were compared to cells derived from postmortem dura mater. In two

50 instances, we have isolated and compared dermal and dural cell lines from the same subject.

51 Notably, striking differences between the dermis and dura mater-derived cell lines were found.

52 Compared to dermal fibroblasts, postmortem dura mater-derived cells demonstrated different

53 morphology, exhibited slower growth rates, failed to express fibroblast protein markers, and

54 exhibited significant differences in gene expression profiles. In addition, dura mater-derived cells

55 were found to exhibit a high rate of chromosomal abnormalities, particularly in the loss of the Y

56 chromosome. Our study highlights potential limitations of postmortem human dura mater-

57 derived cells for disease modeling, argues for rigorous karyotyping prior to reprograming, and

58 brings into question the identity of dura mater-derived cells as belonging to a fibroblast lineage. 


\section{INTRODUCTION}

61 The appeal of human in vitro cell models stems from the potential to recapitulate and elucidate

62 the pathophysiology of numerous diseases, including neurodegenerative disorders. Patient-

63 derived cell lines can address cellular and molecular pathways that complement other models.

64 The utility of patient-derived cell models depends upon their ability to reliably reflect the disease

65 process. For example, a fibroblast patient-derived cell model of Huntington's disease has been

66 reported to clinically recapitulate the disease state better than transformed cellular models [15].

67 In this regard, isolated fibroblasts from routine dermal biopsies are collected in order to be

68 directly differentiated into induced neurons (iNeurons) or reprogrammed into induced pluripotent

69 stem cells (iPSCs) $[6,17]$ that can subsequently be differentiated into neural progenitor cells or

70 different neuronal subtypes. Further incorporation of induced neurons into more complex cellular

71 models, such as three-dimensional brain organoids, represents additional avenues to replicate

72 morphological and functional cell interactions of the human brain [20]. Fibroblasts from dermal

73 biopsies performed in living research subjects have been a common cell source for patient-

74 derived disease models. In certain instances, however, it would also be advantageous to access

75 fibroblasts from postmortem tissue, such as when family members who carry relevant gene

76 mutations are identified or when no other sources are available. For these reasons, an accessible

77 source of postmortem fibroblasts from patients could provide significant value to the field.

78 Human dura mater can provide an alternative source of primary cells that can be directly

79 obtained from postmortem tissue. Dura mater is the outer most layer of the meninges, located

80 between the skull and the brain, and is primarily composed of fibroblasts and collagen [14]. Cells

81 from dura mater can be isolated, cultured, and utilized for several applications. Previous studies

82 have investigated the use of dura mater-derived cells in grafts [9], drug response [10,12], particle 
83 engulfment [18,19], inflammation and healing [5,11], and reprogramming into iPSCs [2,3,22].

84 Brain biobanks oftentimes collect and store dura mater at the time of autopsy. The use of these

85 dura mater-derived cells can be advantageous because they can be studied when other cell

86 sources, such as dermal fibroblasts and peripheral blood mononuclear cells, were not collected

87 during life. Additionally, dura mater-derived cells as an in vitro disease model can provide novel

88 and complementary insights to traditionally established cell-based disease models. Although

89 these dura mater-derived cells have been used in multiple studies, a direct comparison between

90 dermis and dura mater-derived cells has not been reported to our knowledge.

91 In this study, we endeavored to better characterize the cells isolated from postmortem

92 human dura mater to assess their utility as a source of cells for in vitro disease modeling. Sproul

93 and colleagues previously isolated dura mater-derived cells from non-cryoprotected frozen dura

94 mater [22]. Similarly, we isolated cells from postmortem frozen and fresh dura mater from

95 subjects with neurodegenerative diseases and compared cell outgrowth from the tissue, cellular

96 morphology, growth rate, chromosomal karyotype, protein expression of fibroblast markers, and

97 RNA-sequencing profiling to dermis-derived cells. Furthermore, we isolated and characterized

98 dermis and postmortem dura mater-derived cell lines generated from the same subjects. This

99 direct, comparative analysis between cells isolated from the dermis and dura mater highlights the

100 utility and potential limitations of postmortem dura mater-derived cells as an in vitro patient-

101 based disease model.

102

103 MATERIALS AND METHODS

104 Tissue collection 
105 Dermal tissue was provided by the University of California, San Francisco, Memory and Aging

106 Center, where participants enrolled in observational research studies underwent skin biopsy after

107 providing written informed consent. Approximately two-millimeter dermal punches were

108 performed at the inner thigh. The tissue was immersed in complete media composed of DMEM

109 high glucose with sodium pyruvate (Thermo Fisher Scientific, Waltham, MA, USA, \#11995073),

$11010 \%$ heat inactivated FBS (VWR, Radnor, PA, USA, \#97068-091), and 1\% Penicillin-

111 Streptomycin (Thermo Fisher Scientific, \#15140122). Cells were isolated within eight hours

112 after procurement.

113 Postmortem human dura mater was provided by the University of California, San

114 Francisco, Neurodegenerative Disease Brain Bank. Consent for brain donation was obtained

115 from all subjects or their surrogates, following the principles outline in the Declaration of

116 Helsinki. Prior to brain extraction, the overlying dura mater was removed and stored surrounded

117 by wet ice during transport. Upon arrival to the laboratory, a small patch of dura ranging in

118 maximum dimension from two to five centimeters, was dissected and either (1) immersed in

119 complete media in a centrifuge tube or (2) rapidly frozen and banked. The freshly captured dural

120 tissue was stored at $4 \mathrm{C}$, and cells were isolated within 24 hours after procurement. For frozen

121 dural tissues, samples were placed in a freezer bag and surrounded by dry ice, then stored in a -

$12280^{\circ} \mathrm{C}$ freezer for up to 4 years of long-term without the use of a cryoprotectant reagent. The dural

123 tissue was briefly thawed and cells were isolated.

\section{Cell culture}

126 Following tissue collection, both dermal and dural tissues were washed in sterile Dulbecco's 127 phosphate buffered saline (DPBS) without calcium and magnesium (Thermo Fisher Scientific, 
$128 \#$ \#14190250) four times, one minute per wash, in a cell culture dish. The tissue was then

129 transferred into a new dish with complete media. The tissue was divided into smaller sections,

130 approximately one to two millimeters, with surgical tools and transferred into a six-well cell

131 culture plate. A glass coverslip was gently placed over the tissue and complete media was added

132 into the wells. For the remaining fresh dural tissue, it was cryopreserved in complete media and

$13310 \%$ DMSO for long-term tissue storage in liquid nitrogen. Cultures were incubated in a

134 humidified chamber at $37^{\circ} \mathrm{C}$ and $5 \% \mathrm{CO}_{2}$, left undisturbed for the first week, then fed every two

135 to three days with complete media. After approximately three weeks in culture, the cells were

136 collected, expanded, and banked in liquid nitrogen with complete media and 10\% DMSO.

137 To optimize the recovery of cells from frozen dural tissue, an alternative cell culture

138 media was used, composed of DMEM high glucose with sodium pyruvate, $10 \%$ heat inactivated

139 FBS, 1X NEAA (Thermo Fisher Scientific, \#11140050), 1X Glutamax (Thermo Fisher

140 Scientific, \#35050061), $0.1 \mathrm{mM}$ 2-Mercaptoethanol (Thermo Fisher Scientific, \#21985023), 1X

141 Nucleosides (MilliporeSigma, Burlington, MA, USA, \#ES-008-D), and 1\% Penicillin-

142 Streptomycin [3, 22]. This DMEM media with nucleosides was used for the first week in culture

143 and then switched to complete media with the addition of Glutamax and 2-Mercaptoethanol.

144 Alternatively, DMEM media with nucleosides was used throughout the entire time in culture.

146 Immunofluorescence

147 Cell lines were cultured and plated in a four-well cell culture chamber slide (Thermo Fisher

148 Scientific, \#177437). Cell lines included a dermis and frozen dura mater-derived cell line from

149 the same subject and a fresh dural cell line. Upon confluency, cells were washed twice with

150 DPBS with calcium and magnesium (MilliporeSigma, \#D8662), fixed with 4\% 
151 paraformaldehyde (Electron Microscopy Sciences, Hatfield, PA, USA, \#15714) for 20 minutes,

152 washed with $\mathrm{DPBS}^{+/+} 3$ times for 5 minutes each, and then permeabilized with $0.1 \%$ Triton X-

153100 (MilliporeSigma, \#T8787) for 10 minutes. Cells were washed with $\mathrm{DPBS}^{+/+}$and blocked

154 with $1 \%$ bovine serum albumin (Thermo Fisher Scientific, \#BP1605-100) and 0.25\% Triton X-

155100 for 30 minutes. Cells were then incubated with $1 \mathrm{X}$ rhodamine phalloidin conjugated to

156 TRITC (Thermo Fisher Scientific, \#R415) in block solution for one hour and washed with

$157 \mathrm{DPBS}^{+/+}$. All solutions were made in $\mathrm{DPBS}^{+/+}$and performed at room temperature. Slides were

158 mounted with Prolong Gold Antifade Reagent with DAPI (Cell Signaling Technologies,

159 Danvers, MA, USA, \#8961) as a counterstain and imaged on the DMi8 confocal platform (Leica

160 Microsystems, Wetzlar, Germany) with a 10x objective.

161

\section{Cell proliferation}

163 Dermis and dura mater-derived cell lines were plated at the same seeding density in multiple 24-

164 well cell culture plates at day 0 . Over the span of six days, cells were counted every day to assess

165 proliferation. Cells were washed with $\mathrm{DPBS}^{-/-}$, trypsinized with $0.05 \%$ Trypsin-EDTA (Thermo

166 Fisher Scientific, \#25300062), and collected for cell counting. All cell counts were determined

167 utilizing the Bio-Rad TC20 automated cell counter (Bio-Rad Laboratories, Hercules, CA, USA,

168 \#1450102) with a 1:1 dilution of trypan blue solution (Thermo Fisher Scientific, \#15250061) to

169 cell suspension. All cell counts were performed in triplicates.

\section{Chromosomal karyotyping and mycoplasma analysis}

172 For karyotype analysis, dermis and dura mater-derived cell lines were cultured in a T-25 cell

173 culture flask and shipped live to Cell Line Genetics (Madison, WI, USA) for karyotype analysis 
174 using G-banding. For mycoplasma detection, cells were collected, washed with DPBS ${ }^{-/-}$, and

175 DNA was extracted using the QuickExtract DNA Extraction Solution (Epicentre, Madison, WI,

176 USA, \#QE09050) according to the manufacturer's instructions. Mycoplasma PCR detection was

177 performed according to the manufacturer's instructions (Bulldog Bio, Portsmouth, NH, USA, 178 \#25234). All dura mater-derived cell lines were negative for mycoplasma contamination (data 179 not shown).

\section{Western blot}

182 Dermis and dura mater-derived cell lines were collected, washed with DPBS ${ }^{-/}$, and lysed in cold 183 RIPA buffer (Thermo Fisher Scientific, \#89900) with protease (MilliporeSigma, \#4693124001) 184 and phosphatase (MilliporeSigma, \#4906837001) inhibitors. Lysates were spun and the 185 supernatants were collected for western blot analysis. Protein concentration was determined by 186 bicinchoninic acid assay according to the manufacturer's instructions (Thermo Fisher Scientific, 187 \#23225). Samples were denatured, separated on a NuPAGE 4-12\% Bis-Tris protein gel (Thermo 188 Fisher Scientific, \#NP0336BOX), and transferred onto a nitrocellulose membrane. Membranes 189 were blocked in Odyssey blocking buffer (LI-COR, Lincoln, NE, USA, \#927-50000) for one

190 hour at room temperature, incubated with primary antibodies overnight at $4 \mathrm{C}$, and incubated 191 with the appropriate LI-COR secondary antibodies for one hour at room temperature. 192 Membranes were imaged on the LI-COR Odyssey CLx imaging system. The following primary 193 antibodies were used: Rabbit anti-Vimentin (1:1,000, Cell Signaling Technologies, \#5741S), 194 Rabbit anti-S100A4 (1:1,000, Cell Signaling Technologies, \#13018S), and Mouse anti-Actin 195 (1:5,000, MilliporeSigma, \#MAB1501). The following secondary antibodies were used: 680RD 
Donkey anti-Rabbit (1:10,000, LI-COR, \#926-68073), 800CW Donkey anti-Rabbit (1:10,000,

197 LI-COR, \#926-32213), and 800CW Donkey anti-Mouse (1:10,000, LI-COR, \#926-32212).

199 RNA-sequencing

200 From the same subject, fresh dermal and frozen dural cell lines were plated into a six-well cell

201 culture plate and isolated for RNA-sequencing. Cells were trypsinized, washed with $\mathrm{PBS}^{-/-}$, and

202 total RNA was extracted and purified using the Quick-RNA Microprep Kit (Zymo Research,

203 Irvine, CA, USA, \#R1051). Samples were extracted in quadruplicates and RNA was quantified

204 using a NanoDrop. Libraries for RNA-sequencing were prepared using the SMARTer Stranded

205 Total RNA-Seq Kit v2 Pico Input Mammalian library preparation protocol (Takara Bio USA,

206 Inc., Mountain View, CA, USA, \#634413), analyzed on Agilent TapeStation 4200 (Agilent

207 Technologies, Inc., Santa Clara, CA, USA, \#G2991AA), and pooled before paired-end 150-base-

208 pair sequencing on one lane of an Illumina NovaSeq 6000 sequencer (Illumina, Inc., San Diego,

209 USA), which generated 3 million reads per samples. Salmon (v0.13.1) was used to map reads to

210 the human transcriptome (Gencode v28). Tximport (v1.14.0) was used to import the data into $\mathrm{R}$

211 (v3.6.1) and DESeq2 (v1.26.0) was then used to perform differential gene expression analysis.

212 For predicting the target pathways, iPAGE (v1.0) with default settings was used [13], which uses

213 the concept of mutual information to directly quantify the dependency between the expression

214 data set and known pathways in MSigDB.

\section{Statistical analysis}


217 Statistical analysis, t-test, linear regression, and odds ratios with a Fisher's exact test were

218 performed on GraphPad Prism 8. Mean and standard error were reported. P-values less than or

219 equal to $\square 0.05$ were considered significantly different.

\section{$221 \quad$ RESULTS}

\section{The freeze-thaw process decreases cell outgrowth from postmortem dura mater}

223 To investigate the potential of postmortem dura mater as a viable source of cultured fibroblasts,

224 we performed a systematic comparison of the ability to culture cells from two different sources,

225 dermis and dura mater. Dermal cells were obtained from skin biopsies during life and dura mater

226 was either processed shortly after autopsy (fresh) or from banked frozen dura mater (frozen).

227 For this comparison, we processed a total of 77 dermal biopsies for fibroblast cell

228 banking [23]. These samples were obtained from subjects during life with a mean age of $53.6 \pm$

22914.2 years and with more female subjects than male subjects, $62 \%$ and $38 \%$ respectively (Table

230 1). Most subjects were diagnosed with neurogenerative disease, either clinically or through

231 neuropathological assessment (Supplemental Table S1). All processed dermal cell lines produced

232 cell outgrowth, and cell lines were successfully expanded and banked in liquid nitrogen (Table

233 2). Thus, the generation of cell lines from dermal biopsies performed in living subjects was

234 robust and consistent.

235 To compare the success rate of cell outgrowth from dura mater, we utilized the same

236 isolation protocol as for dermal cell banking. We processed a total of 43 fresh dural cases, from

237 subjects with a mean age of $74 \pm 9.3$ years, mean postmortem interval (PMI) of $11.1 \pm 7.6$ hours,

238 and with a slightly greater number of male subjects than female subjects (53\% and $47 \%$

239 respectively, Table 1). Most subjects were diagnosed with neurogenerative disease through 
240 neuropathological assessment (Supplemental Table S1 and Table S2). Forty cases had cell

241 outgrowth that were successfully banked (93\%, Table 2). Furthermore, two dural cases that

242 initially had no cell outgrowth were recovered by reprocessing tissue that was cryoprotected with

243 DMSO and stored in liquid nitrogen post-autopsy. Three fresh dural cases displayed no

244 outgrowth, despite extensive time in culture ( 40 days). In summary, cell outgrowth, generation,

245 and banking from fresh dural tissue were comparable to the dermal tissue.

246 In addition, we investigated the ability to culture cells from banked frozen dura mater. A

247 total of 14 frozen cases were processed, with the mean age of subjects being $66 \pm 8.5$ years,

248 mean PMI of $7.4 \pm 2.2$ hours, and male and female subjects were equally represented (Table 1).

249 Only two frozen dural cases had cell growth that was sufficient for successful banking (14\%,

250 Table 2). Three additional dural cases had limited cell outgrowth, slow proliferation, and poor

251 cell morphology and therefore could not be successfully banked. In an attempt to increase cell

252 recovery from frozen dura mater, we utilized several alternative culture media for seven frozen

253 dural cases $[3,22]$. Despite up to 40 to 70 days in culture, cell outgrowth from the different types

254 of media compositions and combinations could not be consistently improved. Overall, the fresh

255 dural tissue showed significantly better cell outgrowth compared to frozen dural tissue (with an

256 odds ratio of 80, 95\% CI [10.71-407.9], p<0.0001****, Fisher's exact test).

258 Dura mater-derived cell lines exhibit slower outgrowth and proliferation, as well as

259 abnormal morphology

260 As dermis and dura mater-derived cell lines were cultured and banked, differences in initial cell

261 outgrowth from the tissue, cell morphology, and proliferation rates became apparent. From

262 dermal tissue, cell outgrowth was observed by day seven post-dissection and an adequate number 
263 of cells were expanded for cryopreservation after thirty days. In contrast, cell outgrowth from

264 fresh dura mater was observed approximately nine days post-dissection and cells were

265 cryopreserved after thirty-one days. Finally, cells derived from frozen dural tissue took 19 days

266 for outgrowth post-dissection and were cryopreserved after 55 days (Fig. 1). Thus, initial cell

267 outgrowth and banking from dural tissue took longer compared to dermal tissue.

268 Differences in cell morphology between the dermis and dura mater-derived cell lines

269 were also noted. Brightfield images demonstrated that dermis-derived cells exhibited classical

270 fibroblast morphology, with long, spiny, and tightly packed cell bodies. Dural cells derived from

271 fresh dura mater appeared to have an intermediate phenotype, with some normally spindle-

272 shaped cell bodies that were densely packed and others with a more cobblestone shape and

273 disorganized alignment. In contrast, dural cells derived from frozen dura mater displayed

274 abnormal morphological features, such as enlarged cell bodies, decreased uniformity, and

275 decreased cell density (Fig. 2A). Rhodamine phalloidin staining for actin filaments further

276 illustrated the decreased organization of dura mater-derived cell lines (Fig. 2B). Taken together,

277 these observations show differences in cell morphology and arrangement between dermis and

278 dura mater-derived cells, suggesting potential abnormalities in the dura mater-derived cell lines.

279 Next, we documented proliferation rates of several lines. Compared to dura mater-

280 derived cell lines, dermis-derived cell lines resulted in more total cells counted over the span of

281 six days (Fig. 3A). Proliferation rate of the cell counts showed a significant decrease in dura

282 mater-derived cell lines compared to dermis-derived cell lines (Fig. 3B). Overall, dermis-derived

283 cell lines were observed to proliferate about three times faster than dura mater-derived cell lines.

285 Dura mater-derived cell lines exhibit chromosomal abnormalities 
As an important quality control in cell banking, we next performed chromosomal karyotyping to assess gross genetic changes or anomalies in the derived cell lines. From the thirty-six dermisderived cell lines that underwent karyotype analysis, thirty-one cell lines exhibited a normal karyotype (86\%) and five cell lines exhibited an abnormal karyotype (14\%). In contrast, from the twenty-nine fresh dura mater-derived cell lines that underwent karyotype analysis, twelve cell

291 lines exhibited a normal karyotype (41\%), thirteen cell lines exhibited an abnormal karyotype 292 (45\%), and four cell lines exhibited an unstable karyotype (14\%). Of note, the two cell lines

293 derived from frozen dura mater that were successfully banked both exhibited normal karyotypes

294 (Table 2). Overall, dura mater-derived cell lines revealed significantly more chromosomal 295 instability than dermis-derived lines.

297 dura mater-derived cell lines that exhibited abnormal karyotypes, 12 cell lines were derived from 298 male subjects (92\%). Furthermore, the majority of male-derived lines with chromosomal 299 abnormalities exhibited the clonal loss of chromosome Y (LOY). There were eight cell lines that 300 exhibited only LOY, three cell lines that exhibited LOY with additional autosomal abnormalities, 301 and one cell line that exhibited trisomy in chromosome 7 (Table 3). In summary, there were 302 more chromosomal abnormalities in male-derived dural cell lines compared to female-derived 303 dural cell lines (with an odds ratio of 16.8, 95\% CI [1.615-201.2], $\mathrm{p}=0.0112 *$, Fisher's exact 304 test).

\section{Dura mater-derived cell lines fail to express key fibroblast markers}


309 (Supplementary Table S3). We assessed cell identity by immunostaining against two classical

310 markers of fibroblast identity, the intermediate filament protein vimentin and the fibroblast-

311 specific protein 1 (FSP1 or S100A4), a member of the S100 calcium binding family [16] (Fig.

312 4A). While dura mater-derived cells expressed vimentin, albeit at lower levels, they strikingly

313 lacked expression of S100A4 (Fig. 4A-C). In this analysis, we were able to compare protein

314 expression in two pairs of dermis and dura mater-derived cells from the same subjects (Figure

$3154 \mathrm{~A}$, lanes $1 \mathrm{~A} / 1 \mathrm{~B}$ and 2A/2B). Notably, in these "matched" cell lines, levels of the S100A4

316 fibroblast marker appeared decreased in the cells derived from postmortem dura mater compared

317 to cells derived from dermis. Therefore, two fibroblast markers differed even between dermis

318 and dura mater-derived cell lines, even when the cell lines were generated from the same subject.

320 Dermis and dura mater-derived cells from the same subject exhibit highly divergent gene

321 expression profiles

322 To better understand how cells cultured from dermal and dural tissues compare in an unbiased

323 manner, we investigated their transcriptional profiles by RNA-sequencing. For this analysis, we

324 utilized dermis and dura mater-derived cells from the same subject (lanes 1A and 1B from Fig.

325 4A). Notably, by definition, these cells were isolated at two different points in time. The dermal

326 biopsy occurred during life when the subject was age 48 and the dura mater was frozen at the

327 time of autopsy at age 51. RNA-sequencing analysis revealed approximately 3,000 genes that

328 were significantly differentially expressed between the dermis and dura mater-derived cell lines.

329 Gene ontology-term enrichment analysis revealed categories of genes significantly upregulated

330 in dura mater-derived cells involved in cell adhesion, cell migration and proliferation,

331 extracellular matrix and structure, filaments, and signaling. Genes associated with fibroblast 
332 identity that were upregulated in dura mater-derived cells included ACTA2, ITGA1, and P4HA1.

333 Significantly downregulated genes were also found in the same categories: cell adhesion, cell

334 migration and proliferation, extracellular matrix and structure, and filaments. Fibroblast

335 associated genes that were downregulated in dura mater-derived cells included FAP and ANPEP

336 (Fig. 5A, Additional File 1).

337 Given the number of differentially expressed genes, we further investigated implicated 338 molecular pathways between the dermis and dura mater-derived cell lines through iPAGE and

339 gene-set enrichment analyses. This analysis revealed upregulated pathways in categories such as

340 "Regulation of wound healing", which included cell shape, "I-band" which included actin

341 filament, binding, and organization, and "Embryonic skeletal system development" which

342 included cell fate specification, axis elongation, and mesenchymal to epithelial transition. iPAGE

343 analysis also showed dysregulation in "Homophilic cell adhesions via plasma membrane" which

344 included cell to cell adhesion and contact zone, "Potassium ion transmembrane transporter"

345 which included actin filament movement, and "Growth factor activity" which included cell

346 proliferation (Fig. 5B). Together, these data suggest that the dura mater-derived cells

347 significantly differ from dermis-derived fibroblasts in both protein and gene expression, despite

348 being derived from the same subject.

350 Discussion

351 Fibroblast-like cells isolated from dura mater have been generated and reprogrammed into iPSCs

352 and differentiated into neurons [2,3,22]. Postmortem dura mater-derived cells are advantageous

353 when no other cell source is available. With the hope of advancing dura mater-derived cells as a

354 potential cell source for precision medicine applications, we undertook a systematic comparison 
355 between cells isolated from dermal biopsies during life versus postmortem dura mater, either

356 fresh from autopsy or after a period of being frozen. Earlier studies have characterized dura

357 mater cells either collected perioperatively [3] or from postmortem tissue [2, 22]. In the latter

358 cases, the dura mater cells were compared to cells derived from postmortem scalp dermis [2,22].

359 In contrast, this study compared postmortem dural cells to dermal fibroblasts collected during

360 life. A comparison of the study materials, conditions used, and results reported in this and the

361 prior dura mater cell studies can be found in 'Additional file 2'. Our results revealed substantive

362 differences between dermis and dura mater-derived cell lines in cell outgrowth, proliferation,

363 morphology, karyotype, protein expression and transcriptional profiles. Taken together, these

364 results raise questions regarding the "fibroblast" identity of dura mater-derived cells.

365 Some of the differences between our results and those of other groups may be due to

366 technical differences. For example, Sproul et al. reported a higher success rate of fibroblast

367 outgrowth from dura mater, which was processed by freezing in a liquid nitrogen vapor

368 sandwich, with the tissue first chilled between aluminum plates and then placed in liquid

369 nitrogen vapor for five minutes [22]. In comparison, we froze dura mater using a dry ice

370 sandwich method, which is significantly warmer than liquid nitrogen vapor [24]. With these

371 freezing conditions and the lack of a cryoprotectant reagent to prevent ice damage, the frozen

372 dura mater and dural cells may have been damaged, resulting in the unsuccessful establishment

373 of working cell lines or altered morphology. Therefore, careful storage precautions should be

374 taken into consideration while freezing dura mater without a cryoprotectant reagent.

375 Cell outgrowth issues were ameliorated in dura mater that was processed fresh, without

376 being frozen. Despite this, cell lines from fresh dura mater still contained a higher percentage of

377 chromosomal abnormalities when compared to dermal fibroblasts. This observation could stem 
378 from several possibilities, including postmortem tissue source, age of subject, PMI, or more

379 advanced stage of neurodegenerative disease. A significant proportion of the chromosomal

380 abnormalities in dura mater-derived cell lines were from male cases that exhibited LOY.

381 Interestingly, it has been previously reported that LOY in blood cells was associated in aging

382 men with diseases, such as cancer and Alzheimer's disease [8]. This may suggest that

383 postmortem dura mater-derived cell lines from subjects with neurodegenerative diseases have

384 chromosomal instability in vitro.

385 Sproul et al. also previously reported LOY in a dura mater-derived iPSC line which they

386 attributed to in vitro culturing because the Y chromosome was present in the original frozen dura

387 mater through $A M G$ gene amplification [22]. This may be the case here as well. Although,

388 another study generated a cell line from dura mater obtained during surgery and generated an

389 iPSC line with a normal male karyotype [3]. Furthermore, it has been previously reported that

390 derived human stem cells with chromosomal abnormalities have a cellular growth advantage in

391 vitro, which may be similar within the dura mater-derived lines. The mechanisms of

392 chromosomal abnormalities are unclear [1].

393 While karyotype abnormalities appear to be a significant concern for dura mater-derived

394 cells, dural cell lines with a normal karyotype nonetheless did not exhibit a clear fibroblast

395 identity based on protein markers and RNA-sequencing profile expression. Expression

396 differences in cell lines derived from dermis and dura mater may be due to the different tissue

397 types and anatomical locations as has been reported for fibroblasts isolated from different body

398 regions such as the arm or thigh [4,7]. Others have reported a lack or decrease of S100 family

399 protein expression in dura mater-derived cells [5,21,22], similar to what we have shown here.

400 Thus, our data suggest that cells cultured from dura mater may not be fibroblasts but rather an 
401 alternative, yet unidentified cell type. As such, use of dermal cells for disease modeling should

402 proceed with caution.

403

\section{Conclusions}

405 We aimed to assess the possibility of utilizing postmortem dura mater, from both frozen and

406 fresh storage conditions, as a source of in vitro disease cell models. Contrary to our expectations,

407 this study revealed multiple phenotypic, karyotypic, and transcriptional differences between

408 dermis and dura mater-derived cells, even when those cells originated from the same subject.

409 The reasons underlying these differential features are not entirely clear. However, this study

410 should prompt, at a minimum, careful karyotyping and functional assessment of dura mater-

411 derived cells prior to reprogramming into iPSCs. Additionally, further investigation into the

412 overall utility of dura mater-derived cells as a source for disease modeling is warranted. 


\section{$414 \quad$ Additional Files}

\section{List of abbreviations}

416 PMI: postmortem interval; iPSCs: induced pluripotent stem cells; DPBS: Dulbecco's phosphate

417 buffered saline; KT: karyotype; LOY: loss of chromosome Y; S100A4/FSP1: fibroblast-specific

418 protein 1; rVimentin: recombinant vimentin; rS100A4: recombinant S100A4

420 Ethics approval and consent to participate

421 Consent for brain donation was obtained from all subjects or their surrogates in accordance with

422 the Declaration of Helsinki and the research was approved by the UCSF Committee on Human

423 Research. Dermal biopsies were obtained from subjects with written informed consent and the

424 research was approved by the UCSF Committee on Human Research (\#10-00234).

\section{Consent for publication}

427 Not applicable.

\section{Availability of data and material}

430 Cell lines are available upon reasonable request through the Memory and Aging Center at the

431 University of California, San Francisco. A detailed dura mater-derived cell culture protocol can

432 be found at protocols.io: dx.doi.org/10.17504/protocols.io.8m2hu8e. RNA-sequencing data from

433 this study has been deposited to Gene Expression Omnibus.

\section{Competing interests}

436 The authors declare that they have no competing interests. 


\section{Funding}

438 This study was supported by the Chan Zuckerberg Initiative (to AWK, WWS), Rainwater Tau

439 Consortium (to AWK, WWS), R01CA24098-01 (to HG), and K08AG052648 (to SS). AWK was

440 supported by NIH grants (R01AG059052, R01AG057342, P30AG062422), the Paul G. Allen

441 Family Foundation, the John Douglas French Alzheimer' Foundation, and the Alzheimer's

442 Disease Research Center. The UCSF Neurodegenerative Disease Brain Bank was supported by

443 NIH grants (P01AG019724, P50AG023501), the Bluefield Project to Cure FTD, and the Tau

444 Consortium. The Memory and Aging Center was supported by NIH/NIA P50AG023501 (to

445 BM).

\section{Authors' contributions}

448 ARA, WWS, and AWK planned and designed the study. ARA, KG, CIC, and HG performed the

449 experiments. CGC, ALN and AMK provided tissue and supporting information. SS, LTG, and

450 WWS performed neuropathological assessment. ARA, KG, and HG compiled and analyzed the

451 data. ARA and AWK composed the manuscript. All authors read and approved the final

452 manuscript.

\section{Acknowledgements}

455 The authors thank the members of the Kao and Seeley lab for their helpful discussions and 456 support in the laboratory. The authors thank the members of the CZI Neurodegeneration 457 Challenge Network for their support and feedback. The authors gratefully acknowledge and 458 thank the funding that supported this study. We thank the participants and their families for their 459 contributions to this study and research. 


\section{References}

461 1. Assou S, Bouckenheimer J, De Vos J (2018) Concise Review: Assessing the Genome Integrity

462 of Human Induced Pluripotent Stem Cells: What Quality Control Metrics? Stem Cells 36:814-

46321.

464 2. Bliss LA, Sams MR, Deep-Soboslay A, Ren-Patterson R, Jaffe AE, Chenoweth JG, et al

465 (2012) Use of Postmortem Human Dura Mater and Scalp for Deriving Human Fibroblast

466 Cultures. PLoS One 7:e45282.

467 3. Cary WA, Hori CN, Pham MT, Nacey CA, McGee JL, Hamou M, et al (2015) Efficient

468 Generation of Induced Pluripotent Stem and Neural Progenitor Cells from Acutely Harvested

469 Dura Mater Obtained during Ventriculoperitoneal Shunt Surgery. World Neurosurg 84:1256-

$470 \quad$ 1266.e1.

471 4. Chang HY, Chi JT, Dudoit S, Bondre C, Van De Rijn M, Botstein D, et al (2002) Diversity,

472 topographic differentiation, and positional memory in human fibroblasts. Proc Natl Acad Sci

473 USA 99:12877-82.

474 5. Chong K, Kwon WK, Kim JH, Park YK, Yoon W, Kim JH, et al (2019) Inflammation by

475 activated macrophage-like THP-1 cells increases human dura mater cell adhesion with alteration 476 of integrin $\alpha 2 \beta 1$ and matrix metalloproteinase. J Orthop Res 37:706-16.

477 6. D’Souza GX, Rose SE, Knupp A, Nicholson DA, Keene CD, Young JE (2020) The 478 application of in vitro-derived human neurons in neurodegenerative disease modeling. $\mathrm{J}$ Neurosci 479 Res 00:1-17.

480 7. Foote AG, Wang Z, Kendziorski C, Thibeault SL (2019) Tissue specific human fibroblast 481 differential expression based on RNAsequencing analysis. BMC Genomics 20:1-19.

482 8. Forsberg LA (2017) Loss of chromosome Y (LOY) in blood cells is associated with increased 
483 risk for disease and mortality in aging men. Hum. Genet. 136:657-63.

484 9. Goldschmidt E, Cacicedo M, Kornfeld S, Valinoti M, Ielpi M, Ajler PM, et al (2016)

485 Construction and in vitro testing of a cellulose dura mater graft. Neurol Res 38:25-31.

486 10. Goldschmidt E, Hem S, Ajler P, Ielpi M, Loresi M, Giunta D, et al (2013) A new model for

487 dura mater healing: Human dural fibroblast culture. Neurol Res 35:300-7.

488 11. Goldschmidt E, Ielpi M, Loresi M, D’Adamo M, Giunta D, Carrizo A, et al (2014) Assessing

489 the role of selected growth factors and cytostatic agents in an in vitro model of human dura mater

490 healing. Neurol Res 36:1040-6.

491 12. Goldschmidt E, Rasmussen J, Chabot JD, Gandhoke G, Luzzi E, Merlotti L, et al (2016) The

492 effect of vancomycin powder on human dural fibroblast culture and its implications for dural

493 repair during spine surgery. J Neurosurg Spine 25:665-70.

494 13. Goodarzi H, Elemento O, Tavazoie S (2009) Revealing Global Regulatory Perturbations

495 across Human Cancers. Mol Cell 36:900-11.

496 14. Haines DE (2018) The Meninges. In: Haines DE, Mihailoff GA, editors. Fundamental

497 Neuroscience for Basic and Clinical Applications, 5th Edition. Elsevier, Philadelphia, p. 107-

498 121.e1.

499 15. Hung CLK, Maiuri T, Bowie LE, Gotesman R, Son S, Falcone M, et al (2018) A patient-

500 derived cellular model for Huntington's disease reveals phenotypes at clinically relevant CAG

501 lengths. Mol Biol Cell 29:2809-20.

502 16. Kalluri R, Zeisberg M (2006) Fibroblasts in cancer. Nat Rev Cancer 6:392-401.

503 17. Karch CM, Kao AW, Karydas A, Onanuga K, Martinez R, Argouarch A, et al (2019) A

504 Comprehensive Resource for Induced Pluripotent Stem Cells from Patients with Primary

505 Tauopathies. Stem Cell Reports 13:939-955 
506 18. Papageorgiou I, Abberton T, Fuller M, Tipper JL, Fisher J, Ingham E (2014) Biological

507 effects of clinically relevant CoCr nanoparticles in the dura mater: An organ culture study.

508 Nanomaterials 4:485-504.

509 19. Papageorgiou I, Marsh R, Tipper JL, Hall RM, Fisher J, Ingham E (2014) Interaction of

510 micron and nano-sized particles with cells of the dura mater. J Biomed Mater Res - Part B Appl

511 Biomater 102:1496-505.

512 20. Papaspyropoulos A, Tsolaki M, Foroglou N, Pantazaki AA (2020) Modeling and Targeting

513 Alzheimer's Disease With Organoids. Front Pharmacol 11:1-8.

514 21. Schick B, Wolf G, Romeike BFM, Mestres P, Praetorius M, Plinkert PK (2003) Dural cell

515 culture: A new approach to study duraplasty. Cells Tissues Organs 173:129-37.

516 22. Sproul AA, Vensand LB, Dusenberry CR, Jacob S, Paul Vonsattel JG, Paull DJ, et al (2014)

517 Generation of iPSC lines from archived non-cryoprotected biobanked dura mater. Acta

518 Neuropathol Commun 2:4

519 23. Takashima A. (1998) Establishment of Fibroblast Cultures. Curr Protoc Cell Biol 00:2.1.1-

$520 \quad 2.1 .12$

521 24. Vonsattel JPG, del Amaya MP, Keller CE (2008) Twenty-first century brain banking.

522 Processing brains for research: The Columbia University methods. Acta Neuropathol 115:509-3 
Tables

Table 1 Demographics of dermal and dural cases

\begin{tabular}{llll} 
& Dermis & & Dura mater \\
\hline Mean & Fresh & Fresh & Frozen \\
\hline Number of cases & 77 & 43 & 14 \\
Age (years) & $53.6 \pm 14.2$ & $74 \pm 9.3$ & $66 \pm 8.5$ \\
PMI (hours) & - & $11.1 \pm 7.6$ & $7.4 \pm 2.2$ \\
Sex (M/F) & $29 / 48$ & $23 / 20$ & $7 / 7$ \\
\hline
\end{tabular}


Tables

Table 2 Successful cell outgrowth and karyotype (KT) analysis of dermal and dural cases

\begin{tabular}{llll} 
& Dermis & \multicolumn{2}{c}{ Dura mater } \\
\hline & Fresh & Fresh & Frozen \\
\hline Outgrowth & $77 / 77(100 \%)$ & $40 / 43(93 \%)$ & $2 / 14(14 \%)$ \\
Normal KT & $31 / 36(86 \%)$ & $12 / 29(41 \%)$ & $2 / 2(100 \%)$ \\
Abnormal KT & $5 / 36(14 \%)$ & $13 / 29(45 \%)$ & $0 / 2(0 \%)$ \\
Unstable KT & $0 / 36(0 \%)$ & $4 / 29(14 \%)$ & $0 / 2(0 \%)$ \\
\hline
\end{tabular}


bioRxiv preprint doi: https://doi.org/10.1101/2021.05.17.444554; this version posted May 18, 2021. The copyright holder for this preprint (which was not certified by peer review) is the author/funder. All rights reserved. No reuse allowed without permission.

Tables

Table 3 Chromosomal abnormalities of dural cell lines from fresh dura mater

\begin{tabular}{lll} 
& Male $(\mathbf{n}=\mathbf{1 2})$ & Female $(\mathbf{n}=\mathbf{1})$ \\
\hline Trisomy & 1 & 1 \\
LOY & 8 & - \\
LOY with autosomal abnormalities & 3 & - \\
\hline
\end{tabular}

LOY $=$ Loss of chromosome $\mathrm{Y}$ 
Tables

Table S1 Diagnosis of dermal and dural cases

\begin{tabular}{llll} 
& Dermis & \multicolumn{2}{c}{ Dura mater } \\
\hline & Fresh $(\mathbf{n}=\mathbf{7 7})$ & Fresh $(\mathbf{n}=\mathbf{4 3})$ & Frozen $(\mathbf{n}=\mathbf{1 4})$ \\
\hline Neuropathological diagnoses of disease & $8(10 \%)$ & $38(88 \%)$ & $14(100 \%)$ \\
Clinical diagnoses of disease & $51(66 \%)$ & - & - \\
Clinically normal & $14(18 \%)$ & $5(12 \%)$ & - \\
Biospecimen only & $4(5 \%)$ & - & - \\
\hline
\end{tabular}


Tables

Table S2 Neuropathological diagnosis of all dural cases

\begin{tabular}{ll} 
Neuropathological diagnosis & No. of cases \\
\hline AD & 13 \\
ADNC & 6 \\
AGD & 1 \\
ALS-TDP-43 & 1 \\
CTE & 1 \\
Chronic Wernicke-Korsakoff & 1 \\
Encephalopathy & 19 \\
FTLD-TAU (PSP, CBD, Pick's) & 6 \\
FTLD-TDP-43 (Type A, B, Unclassifiable) & 3 \\
LBD & 1 \\
Motor and sensory neuropathy & 5 \\
Clinically normal & \\
\hline
\end{tabular}

$\mathrm{AD}=$ Alzheimer's disease, $\mathrm{ADNC}=$ Alzheimer's disease neuropathologic change, $\mathrm{AGD}=$ Argyrophilic grain disease, ALS = Amyotrophic lateral sclerosis, CTE $=$ Chronic traumatic encephalopathy, TDP-43 = TAR DNA-binding protein 43, FTLD = Frontotemporal lobar degeneration, LBD = Lewy body dementia 
Tables

Table S3 Selected dermal and dural cell lines for western blot analysis

\begin{tabular}{lllllll}
\hline Lane & $\begin{array}{l}\text { Derived } \\
\text { tissue }\end{array}$ & $\begin{array}{l}\text { Tissue } \\
\text { arrival }\end{array}$ & $\begin{array}{l}\text { Age at } \\
\text { biopsy }\end{array}$ & $\begin{array}{l}\text { Age at } \\
\text { death }\end{array}$ & PMI & Sex \\
\hline 1 & Dermis & Fresh & 72 & - & - & $\mathrm{M}$ \\
2 & Dermis & Fresh & 52 & - & - & $\mathrm{F}$ \\
3 & Dermis & Fresh & 67 & - & - & $\mathrm{F}$ \\
$4^{1 \mathrm{~A}}$ & Dermis & Fresh & 48 & - & - & $\mathrm{F}$ \\
$5^{2 \mathrm{~A}}$ & Dermis & Fresh & 65 & - & - & $\mathrm{F}$ \\
6 & Dura mater & Fresh & - & 72 & 28.2 & $\mathrm{M}$ \\
7 & Dura mater & Fresh & - & 69 & 11.6 & $\mathrm{M}$ \\
8 & Dura mater & Fresh & - & 60 & 20.5 & $\mathrm{~F}$ \\
9 & Dura mater & Fresh & - & 82 & 9.6 & $\mathrm{~F}$ \\
$10^{1 \mathrm{~B}}$ & Dura mater & Frozen & - & 51 & 5.4 & $\mathrm{~F}$ \\
$11^{2 \mathrm{~B}}$ & Dura mater & Frozen & - & 66 & 7.7 & $\mathrm{~F}$
\end{tabular}

Dermal cell line 1A and dural cell line 1B are from the same subject.

Dermal cell line 2A and dural cell line 2B are from the same subject. 
Figures

\section{Figure 1.}

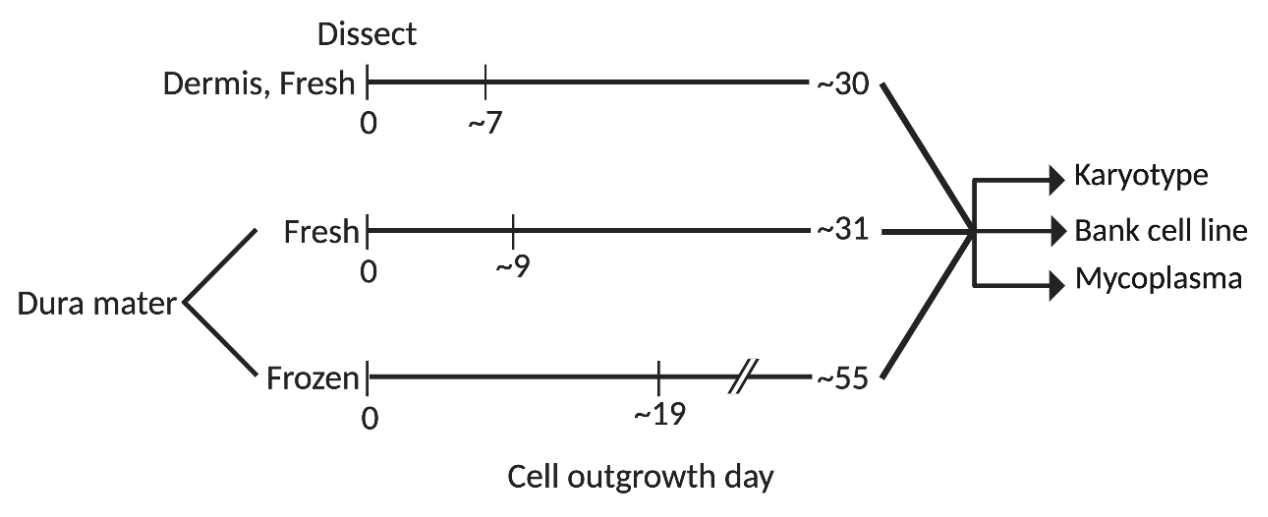

Figure 1. Timeline in days for the isolation, expansion, and banking of dermal cells from fresh dermal and dural cells from fresh and frozen dura mater. Cell outgrowth, proliferation, and banking from fresh and frozen dural tissue were delayed compared to dermal tissue. Successfully banked cell lines were karyotyped and tested for mycoplasma. 


\section{Figure 2.}

A

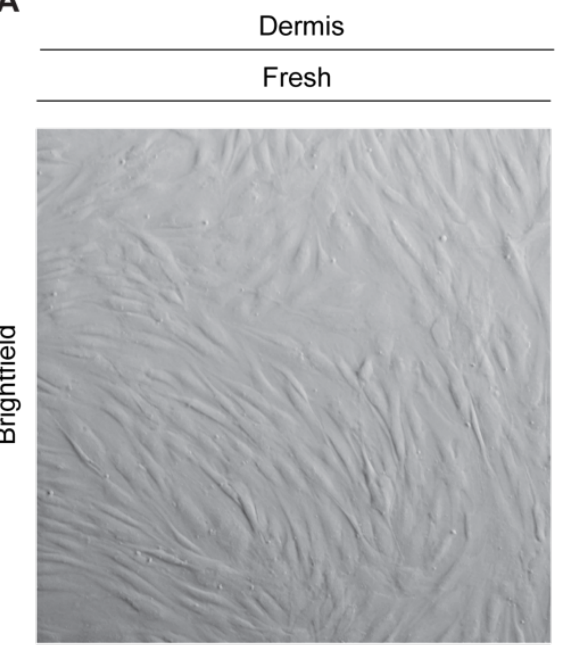

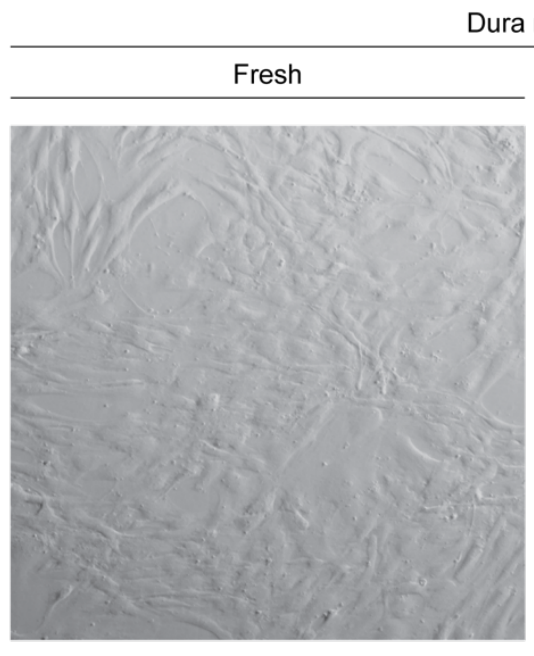

Dura mater

B
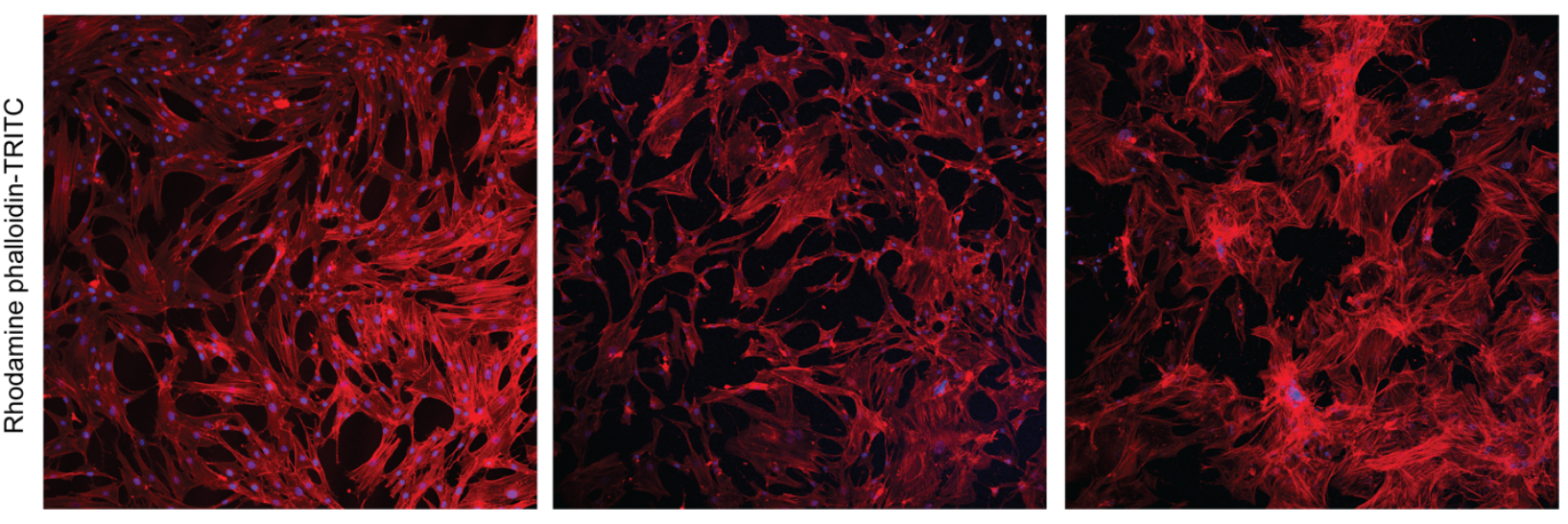

Figure 2. Dural cells displayed reduced cell outgrowth with larger cell body morphology than cultured dermal cells. A Dermal cells exhibit classical fibroblast morphology, with long, spiny, and tightly packed cell bodies. In contrast, dural cells are enlarged, variable, and have disorganized alignment. Dermal and frozen dural cells are from the same subject. Representative brightfield images are shown for each cell type and condition with a 5x objective B Cells were stained with rhodamine phalloidin conjugated to TRITC, reflecting differences in actin filament arrangement between dermal and dural cells. Representative immunofluorescent images are shown for each cell type and condition with a 10x objective. 
Figures

\section{Figure 3.}

A

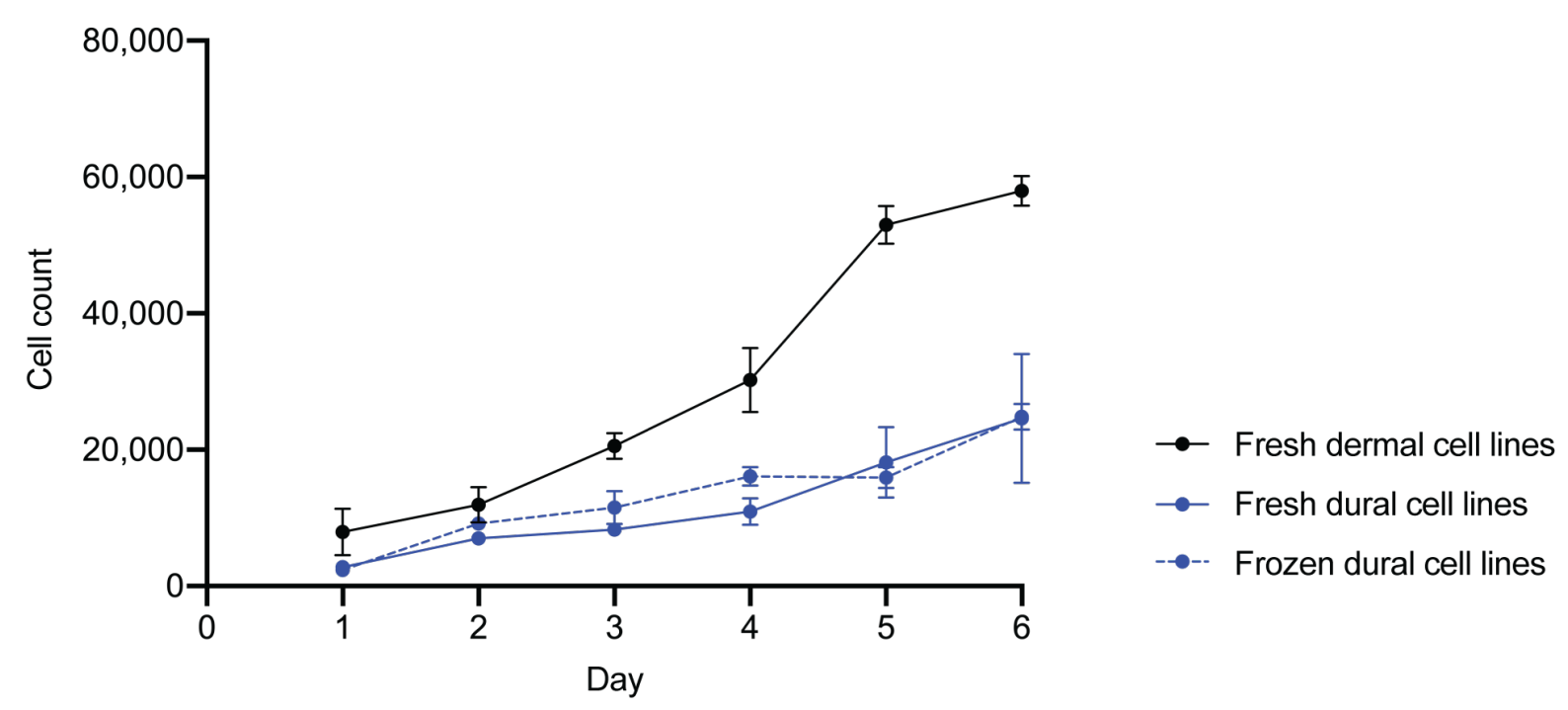

B

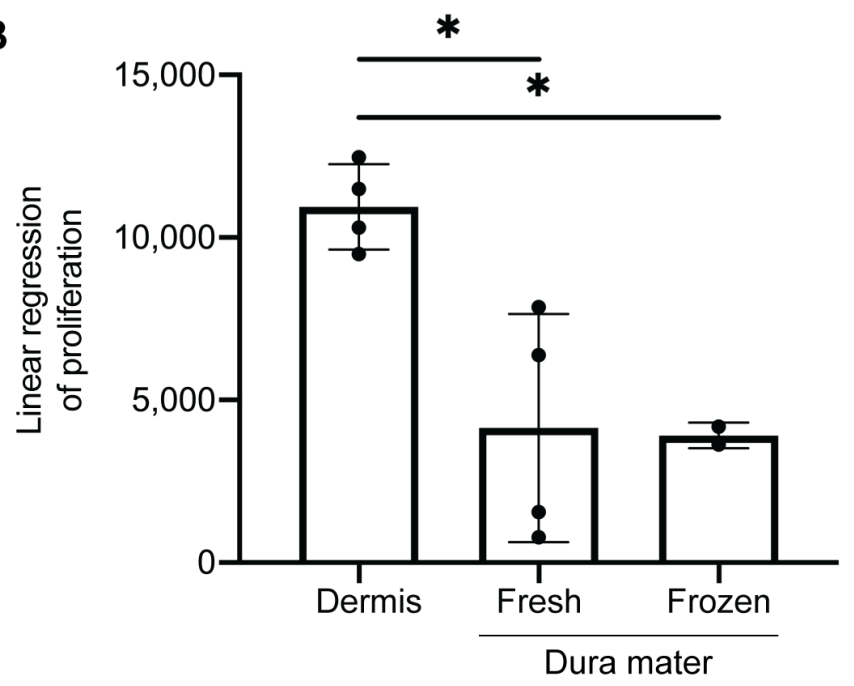

Figure 3. Differences in cell proliferation were observed between cells derived from dermis and dura mater. A Four dermal cell lines (black), four fresh dural cell lines (solid blue), and two frozen dural cell lines (dotted blue) were plated on day 0 and the total number of cells were counted every day for a span of six days. Total cell counts for each day are shown, performed in triplicates. B Cell proliferation rates for dermal and dural cell lines are shown. There was a significant decrease 
bioRxiv preprint doi: https://doi.org/10.1101/2021.05.17.444554; this version posted May 18, 2021. The copyright holder for this preprint (which was not certified by peer review) is the author/funder. All rights reserved. No reuse allowed without permission.

Figures

in cell proliferation in both frozen and fresh dural cell lines compared to the dermal cell lines (One-

way ANOVA, * $\left.{ }^{*}<0.05\right)$. 
Figures

\section{Figure 4.}

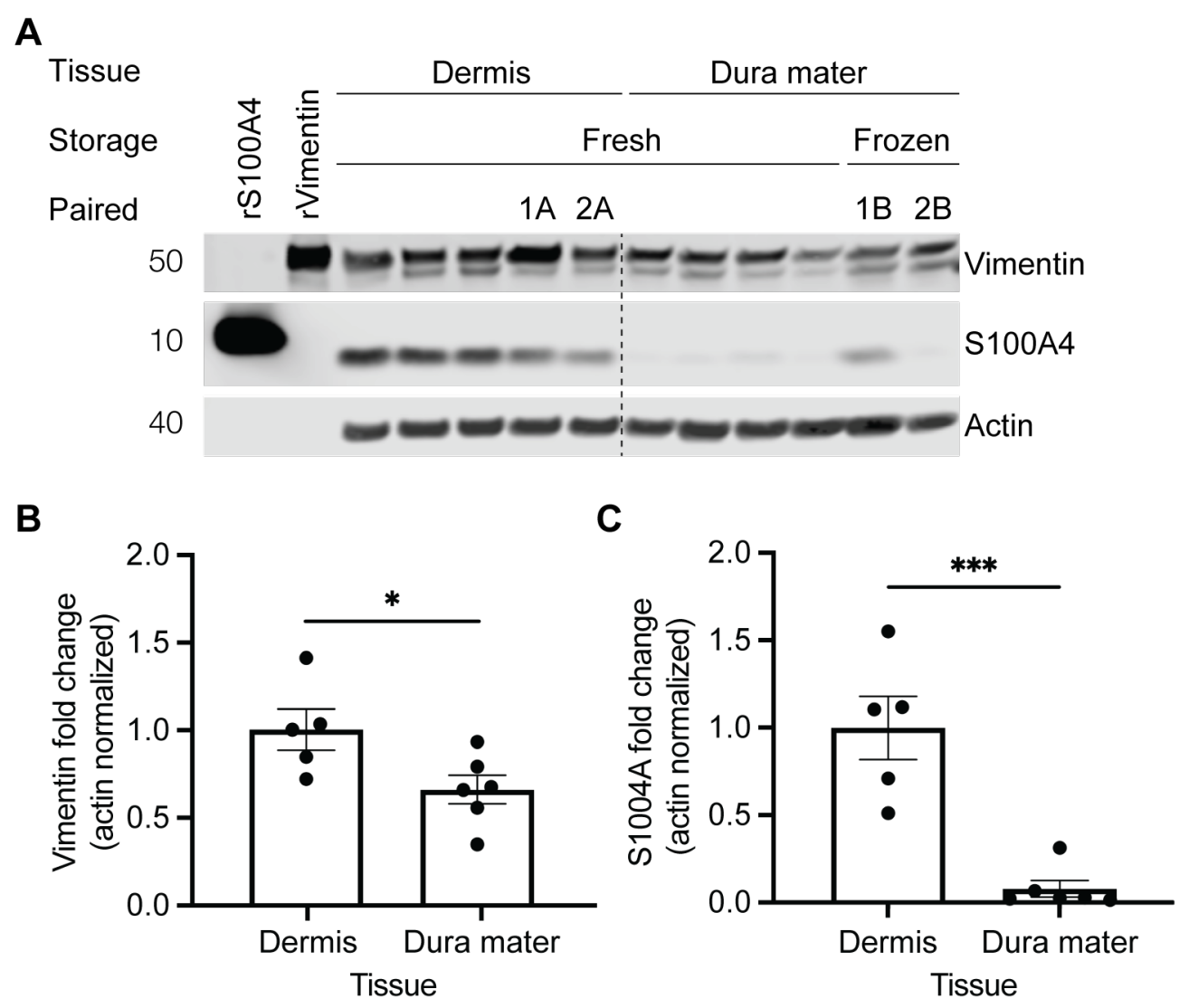

Figure 4. Protein expression differences in fibroblast markers were observed between dermal and dural cell lines. A Cell lysates from five dermal and six dural cell lines $(20 \mu \mathrm{g})$ were immunoblotted with anti-Vimentin and anti-S100A4 antibodies. Tissue indicates from which source the cells were derived from, storage indicates whether tissue underwent frozen or fresh storage conditions before processing, and paired indicates cell lines derived from the same subject ( 2 cases, lanes 1A/1B and 2A/2B). Actin was used as a loading control. rVimentin and rS100A4 were recombinant proteins that were used as positive controls for their respective antibodies. B There was a significant decrease in vimentin protein levels in dural cell lines relative to the dermal 
bioRxiv preprint doi: https://doi.org/10.1101/2021.05.17.444554; this version posted May 18, 2021. The copyright holder for this preprint (which was not certified by peer review) is the author/funder. All rights reserved. No reuse allowed without permission.

Figures

cell lines (normalized to actin, unpaired t-test, $* \mathrm{p}<0.05$ ). $\mathbf{C}$ There was a significant decrease in S100A4 protein levels in dural cell lines relative to the dermal cell lines (normalized to actin, unpaired t-test, $* * * \mathrm{p}<0.0005)$. 


\section{Figure 5.}

\section{A}

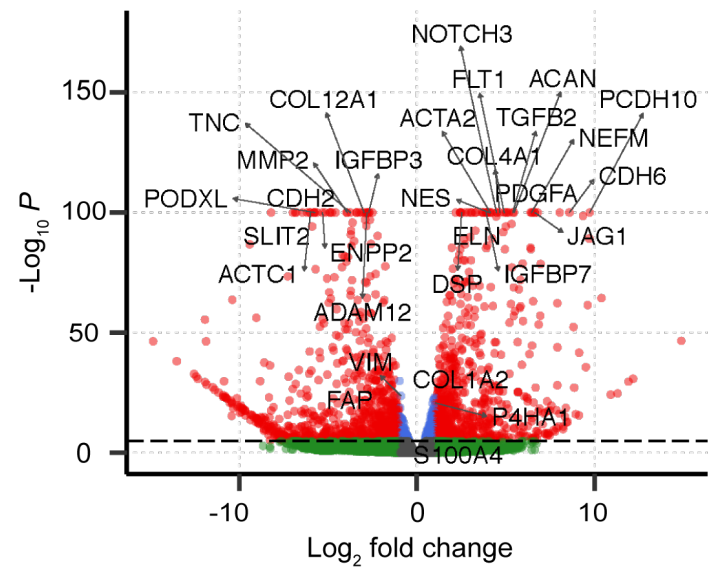

B

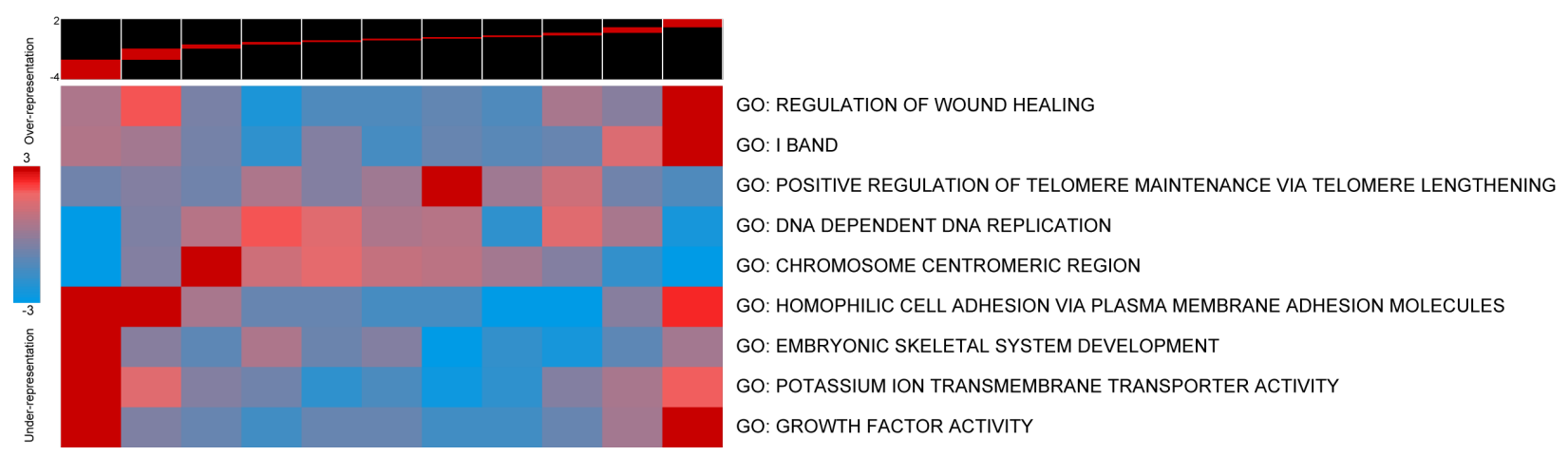

Figure 5. RNA-sequencing analysis of differentially expressed genes in dermal versus dural cells from the same subject. A Volcano plot of differentially expressed genes. Red dots denote differentially expressed genes, blue dots denote genes with expression differences of $-\log _{10} P$ between -1 and 1, and the green dots denote non-significantly expressed genes. B iPAGE pathways representation of differentially expressed genes. Top panel shows expression bins of genes with lower to higher expression in the dural cell line. Heatmap below indicates expression levels relative to GO terms. Red indicates enrichment/over-representation of pathway genes and blue indicates depletion/under-representation. 